\title{
Demonstration of Osseous Tissue in Quail Embryo by Alizarin Red Technique
}

\author{
K. Iniyah", S. Jayachitra, K. Balasundaram and K. Rajendran \\ Department of Veterinary Anatomy, Veterinary College and Research Institute, \\ TANUVAS, Namakkal, Tamil Nadu- 637 002, India \\ *Corresponding author
}

\begin{tabular}{|c|c|}
\hline & A B S T R A C T \\
\hline & \multirow{5}{*}{$\begin{array}{l}\text { The embryonated Namakkal quail eggs were collected from the hatchery at tenth } \\
\text { and fourteenth day of incubation. The eggs were opened by standard technique } \\
\text { and the embryos were collected and stained by alizarin red staining technique to } \\
\text { demonstrate the osseous tissue development. The scapula, coracoid, clavicle and } \\
\text { proximal bones of wing were well developed in both tenth and fourteenth day } \\
\text { embryos. But the fusion of pelvic girdle with the synsacrum and the distal bones } \\
\text { of wing were completely developed only in fourteenth day embryo. The femur, } \\
\text { tibia, fibula, tibiotarsus, tarsometatarsus and digits were found like a rod of bones } \\
\text { without distinct joints in tenth day embryo. Skull bones, vertebral bones and ribs } \\
\text { were fully ossified in fourteenth day quail embryos. But the sternal ossification } \\
\text { was not observed by alizarin red staining technique in both the quail embryos. A } \\
\text { ring of scleral ossicles in the sclera around the margin of cornea were distinctly } \\
\text { observed in both the embryos studied. }\end{array}$} \\
\hline & \\
\hline $\begin{array}{l}\text { Alizarin red, } \\
\text { Osseous tissue, } \\
\text { Quail embryos, } \\
\text { Skeletal } \\
\text { development. }\end{array}$ & \\
\hline Article Info & \\
\hline $\begin{array}{l}\text { Accepted: } \\
\text { 26 May } 2017 \\
\text { Available Online: } \\
\text { 10 June } 2017\end{array}$ & \\
\hline
\end{tabular}

\section{Introduction}

The Quail is a member of the pheasant (Phasianidae) family and in comparison to the chicken, the quail eggs are small and the incubation period is approximately 16.5 days (Ainsworth et al., 2010). Birds resemble other amniotes, in that most of the skeleton is initially laid down in cartilage which subsequently becomes ossified.

The salient characteristics of birds such as fusion of some of the vertebra (synsacrum, pygostyle), presence of scleral ossicles occurs during embryological development are the adaptation to flight in birds (Bellairs and Osmond, 2005). Whole mount staining with alizarin red for osseous tissue has been widely used for visualizing the skeletal patterns of embryos of chicks and small adult vertebrates (Yamazaki et al., 2011). The correct evaluation of mineralization is fundamental for the study of skeletal development. Hence this study was undertaken to achieve the demonstration of bony elements by alizarin red staining. The embryos were stained with alizarin red to reveal the osseous tissue development in quail embryos.

\section{Materials and Methods}

For the study, the embryonated Namakkal quail eggs were collected from the hatchery at tenth and fourteenth day of incubation. The 
eggs were opened by standard technique and the collected embryos were preserved in $10 \%$ neutral formaldehyde solution. Afterwards, the specimens were treated with $95 \%$ alcohol for 3 days. Then they were kept in $1 \% \mathrm{KOH}$ solution for about one month until the calcified areas were clearly visible. The embryos were then transferred to a solution of alizarin red ' $\mathrm{S}$ ' and kept undisturbed until the ossified tissues becomes red (Bancroft and Stevens, 1996). The alizarin red stained embryos were utilized for the study of osseous development in quail (Fig. 1).

\section{Results and Discussion}

\section{Appendicular skeleton}

The pectoral girdle bones viz scapula, coracoid and clavicle were developed fully and appeared red with alizarin red staining technique in both tenth and fourteenth day quail embryos. Hall (2001) stated that the ossification begins at eighth day and completed at tenth day in chick embryos. The proximal bones of the wing, the humerus, radius and ulna were completely ossified at tenth day itself, as mentioned by Yander and Searle (1980) in chick embryo skeletal ossification.

The distal bones of the wing, the carpometacarpus were completely fused with each other in fourteenth day embryo and they were not fused at the extremities in the tenth day quail embryo. In both stage of quail embryos, the phalanges of wing were well developed and clearly separated in each digit (Bellairs and Osmond, 2005).

The three components of pelvic girdle, ilium, ischium and pubis were completely developed in fourteenth day quail embryo but could not be noticed in the tenth day quail embryo. It was also observed that the ilium fused with the synsacrum, the ischium fused with the ventral part of ilium and the pubis fused with the ventral border of ischium in fourteenth day quail embryos (Chevalier, 1977). But in tenth day embryo, the fusion of bones with other was not complete.

In the hind limb skeleton, the femur, tibiotarsus and tarsometatarsus were fully developed like a rod of bones in the day ten of quail embryo without formation of joints between each other. But in the fourteenth day quail embryo, they were completely fused with each other and also formed a distinct joint. Bellairs and Osmond (2005) also reported that the ossification starts after eighth day in chick embryo. In both tenth and fourteenth day of quail embryos, the patella which is a small sesamoid bone in the anterior aspect of knee joint was not clearly ossified. It concurs with the Bellairs and Osmond (2005) who also stated that the ossification of patella does not takes place until about 11 weeks after hatching in chicken.

The digits in the limbs were distinctly formed in both the quail embryos (tenth and fourteenth) and they were clearly separated from each other with distinct joints in fourteenth day embryo and the digits were separated without proper joints in tenth day quail embryo (Panman and Zeller, 2003)

\section{Axial skeleton}

In tenth day quail embryo, all the skull bones were ossified and they were united with somewhat loose attachments. But in fourteenth day quail embryo, all the bones were rigidly fused with each other and given the exact original shape to the skull. Romanoff (1960) also stated that the ossification begins at day ninth and most of the skull bones have undergone ossification by day fourteenth in chick embryo. A ring of scleral ossicles in the sclera around the margin of cornea were clearly observed in both the quail embryos studied. 
Fig.1 Showing ossification of bones with Alizarin red S in tenth

(a) And fourteenth day (b) quail embryo

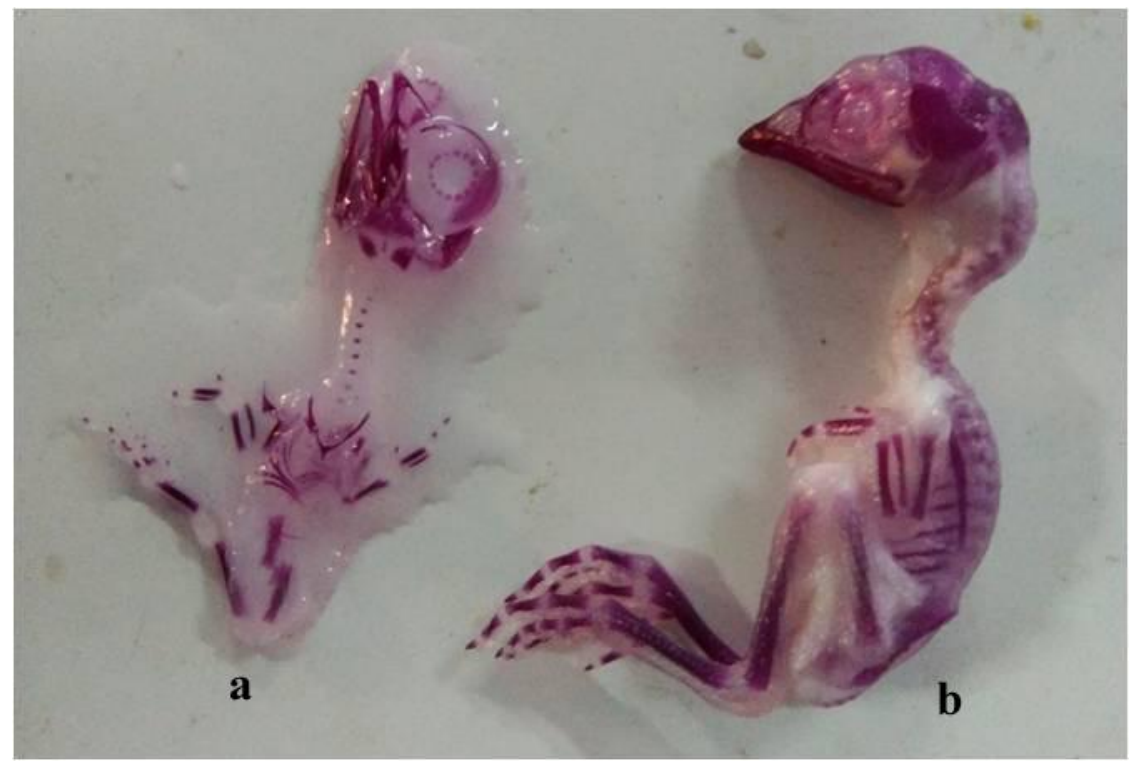

Hall (2001) also stated that the scleral ossification and scleral ossicle were present between day 6.5 and day 10 in chick embryos.

Regarding vertebral bones, the vertebra in each region appeared red stained particularly body of the vertebra and it was found stained throughout the whole length of the vertebral column in tenth day quail embryo.

But in fourteenth day quail embryo all the parts of vertebra in each region were completely ossified and fused with each other as seen in post natal life. Shapiro (1992) mentioned that vertebral bodies are cartilaginous at 5 days and dorsal spines at 6.5 days and by day 8 almost every part of vertebra has become cartilaginous and the ossification completed by hatching in chicks (Bellairs and Osmond, 2005).

The ribs were originated as outgrowth from the vertebra in the neck and trunk region. In the present study, both the stage of quail embryos showed completely ossified ribs. Bellairs and Osmond (2005) stated that the ribs begins to appear at the end of day 6 becomes cartilaginous about 7 to 7.5 days and ossify at 10-12 days.

In both the stage of quail embryos, there was no ossification of sternum observed by alizarin red staining. But, Chevallier (1977) reported that the chondrification of sternum is completed by nine days and ossification begins in the chick embryos at about the time of hatching only.

The present study on demonstration of ossification by alizarin red staining concluded that all the bones of appendicular and axial skeleton except sternum were completely ossified in both tenth and fourteenth day quail embryos. But the joints between bones were very distinctly formed in fourteenth day embryo whereas in tenth day embryo the bones on all over the body were loosely attached to each other without distinct joint regions.

\section{References}

Ainsworth, S.J, Stanley, R.L. and Evans, D.J.R. 2010. Developmental stages of 
the Japanese quail. J. Anat. 216: 3-15.

Bancroft, J.D. and A. Stevens, 1996. Theory and Practice of Histological Techniques.

$4^{\text {th }}$ Edn. Churchill Livingstone, Edinburgh, London.

Bellairs, R. and Osmond, M. 1998. The Atlas of Chick Development. Acad. Press, New York.

Chevallier, A. 1977. Origines des ceintures scapulaires ET pelviennes chez lembryon doiseau. JEEM 423: 275-92.

Hall, B.K. 2001. Development of the clavicles in birds and mammals. J. Exp. Zool. 289: 153-61.

Panman, L. and Zeller, R. 2003. Patterning the limb before and after $\mathrm{SHH}$ signalling. J. Anat. 202: 3-12.
Romanoff, A.L. 1960. The Avian Embryo. Structure and Functional Development. MacMillan, New York.

Shapiro, F. 1992. Vertebral development of the chick embryo during days 3-19 of incubation. J.Morph. 213: 317-33.

Yamazaki, Y., Yugochi, M., Kubota, S. and Isokawa, K. 2011. Whole-mount bone and cartilage staining of chick embryos with minimal decalcification. Biotech Histochem. 86(5): 351-8.

Yander, G. And Searle, R.L. 1980. A scanning electron microscopic study of the development of the shoulder, visceral arches, and the region ventral to the cervical somites of the chick embryo. Am. J. Anat. 157: 27-39.

\section{How to cite this article:}

Iniyah, K., S. Jayachitra, K. Balasundaram and Rajendran, K. 2017. Demonstration of Osseous Tissue in Quail Embryo by Alizarin Red Technique. Int.J.Curr.Microbiol.App.Sci. 6(6): 22862289. doi: https://doi.org/10.20546/ijcmas.2017.606.270 